\title{
A SURVEY OF RADIOLOGY IN CROHN'S DISEASE AND ULCERATIVE COLITIS
}

\author{
Dr HANS HERLINGER, MD, DMRD, DCH, DTM\&H
}

\author{
St James' Hospital, Leeds
}

The plain film should be done before any barium enema examination and is important for three reasons:

a. It will show if the colon contains faecal material and would then be unsuitable for a barium study.

b. It may show evidence of a toxic megacolon, i.e. diameter in excess of $8 \mathrm{~cm}$ together with mucosal surface protrusions into the lumen. This would contraindicate a barium enema examination.

c. It may show evidence of active inflammatory disease, may make possible a limited diagnosis without barium study.

The instant enema

In active disease the inflamed part of the colon is usually free of faecal material, may even be totally contracted and without air. Bowel preparation prior to the barium enema would be both unnecessary and contra-indicated in such patients. A double contrast barium enema without preparation can produce an accurate surface study in the area of active disease.

\section{The barium enema}

Most major departments of radiology in this country employ the so-called double contrast method because of the greater surface detail obtained in this way. In the simple conventional barium enema the colon is filled with barium and may become totally non-translucent to X-rays. Any mucosal surface change will then be visible in tangent only, as a profile view of a limited portion of mucosa. In the double contrast method the lumen is distended with radiolucent material like air, and at the same time the mucosal surface coated with a layer of barium. We now have not only a tangential view of pathology but the mucosa can also be studied en face, and this greatly improves the radiological diagnosis of finer details. The double contrast examination can provide early evidence of pathology and a somewhat more accurate estimation of the extent of a disease process. Moreover, it is a safe examination and no more time consuming than the conventional examination.

In a normal double contrast enema the mucosal surface presents as a straight smooth line of barium coating. Colonic haustrations are well shown but may be absent or diminished in the left colon (Figure 1).

\section{Ulcerative colitis}

In the earlier stages the double contrast enema shows a granular change of the mucosa, giving a mucosal surface outline thicker and more uneven than 
normally. Slightly later in the disease mucosal stippling is seen, an expression of still superficial ulceration covering a large part of the surface. Somewhat later, still in reasonably early disease, there is a slightly coarser irregularity of the mucosal surface, representing superficial ulceration together with oedematous swelling of non-ulcerated mucosa (Figure 2). In the later stages of disease ulceration has spread below the mucosa, producing extensive undermining and at times an appearance of almost continuous ulceration (Figure 3 ).

All these changes, whether early or more advanced, extend from the rectum in a craniad direction, presenting an uninterrupted disease pattern which may, however, show local variations of intensity. Except in circumstances where focal steroid treatment has been given the rectum will always be affected to the same degree. Haustral loss present in addition to the mucosal surface abnormality, is symmetrical and total. In long standing disease the barium enema may show evidence of a stricture or of the development of malignancy.

When the disease process has been present for a year or more inflammatory polyps may appear in 10 to 20 per cent of patients. They can be large or small, few or many, scattered or grouped, rounded or filiform, may even show a linear arrangement across the long axis of the gut.

\section{Crolnn's disease}

The plain film may be helpful. Not only does it show mucosal surface changes but may also give an indication of increased wall thickness. It may be possible to visualise the bowel wall between air outlining its lumen and properitoneal fat against its serosal surface. A normal colon wall does not exceed $2 \mathrm{~mm}$ in thickness while the gut wall in Crohn's disease tends to measure $6 \mathrm{~mm}$ or more (Figure 4).

The earliest changes radiologically visible in Crohn's disease are aphthous ulcerations. They present as fine collections of barium surrounded by a radiolucent halo set within otherwise normal mucosa. They cannot be recognised in profile in a colon filled with barium as in the conventional method. Their recognition is only possible in the double contrast barium enema. In more advanced Crohn's disease we see linear ulcerations, partly transaxial, which can often be followed to the edge of the lumen into clefts which extend into the bowel wall (Figure 5). Numerous linear ulcerations, both transaxial and longitudinal together with the swollen appearance of the non-ulcerated mucosa produce the so-called cobblestone pattern. typical of Crohn's disease. Aphthous ulcerations are frequently seen at the edge of diseased segments between advanced disease and normal mucosa (Figure 6).

A typical feature of Crohn's disease is the unevenness of its distribution. Segments of disease are separated by areas of normal colon. Even with diseased segments the transaxial involvement is uneven and it is possible to see a residual haustra on one side of the colon while on the opposite side they have entirely disappeared (Figure 7). Ulcerations in Crohn's disease tend to be deeper than in ulcerative colitis and present a ragged appearance (see Figure 4). One of the complications of colonic Crohn's disease is fistula formation, a consequence of the transmural disease process (Figure 8). 


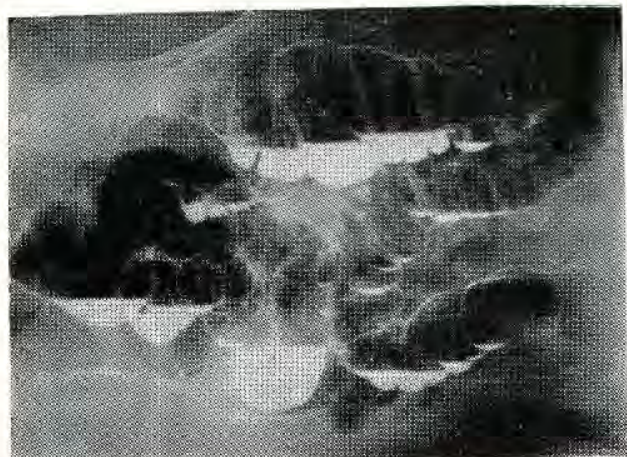

Fig. 1. Lefi lateral decubitus view of a normal double contrast barium enema. Note the smooth thin line of barium coating of the mucosa and the regular outline of the haustrations.

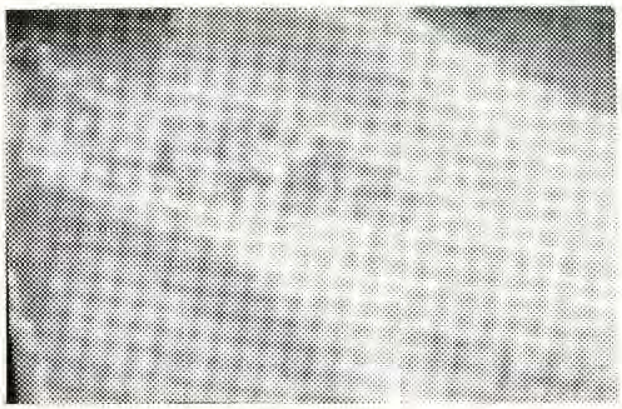

Fig. 3. Advanced ulcerative colitis, sigmoid colon. Ulcerations have undermined the mucosa and give the impression of confluence in the sub-mucosa.

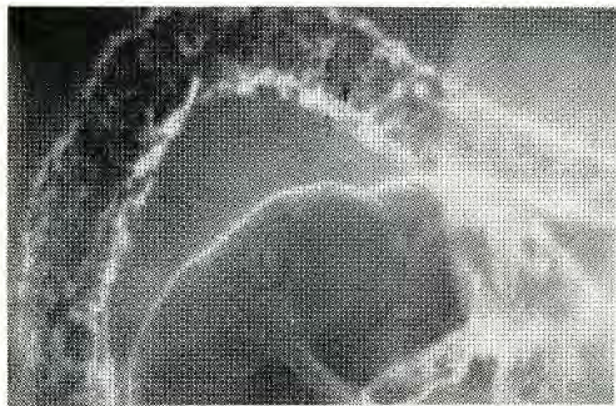

Fig. 5. Crohn's disease of descending colon and the splenic flexure. Extensive linear ulceration with several barium filled lines extending into clefts which project beyond the expected limit of the mucosal surface. Almost normal appearance of the sigmoid colon.

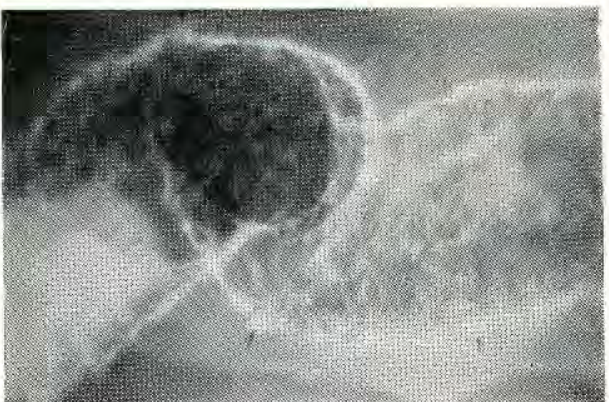

Fig. 2. Ulcerative colitis in the descending colon. Fine ulcerations are widespread, intervening mucosa shows a swollen surface appearance.

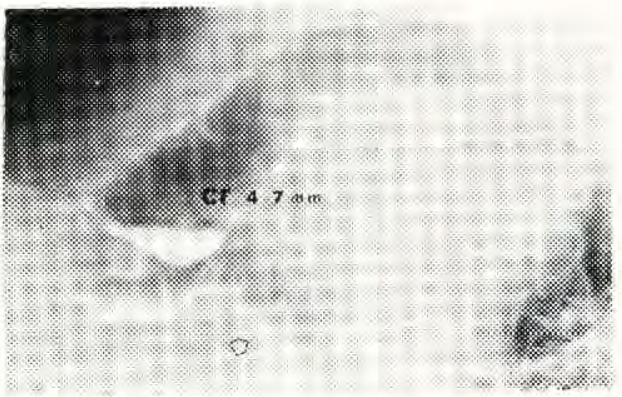

Fig. 4. Barium enema in the right lateral decubitus. Crohn's disease with ulcerations of the transverse colon (arrowed). The descending colon is less severly involved but shows mural thickening, visible as soft tissue density between the barium/air outlined mucosa and the properitoneal fat outlined serosa. Wall thickness measures up to $7 \mathrm{~mm}$, well in excess of normally and in keeping with Crohn's disease.

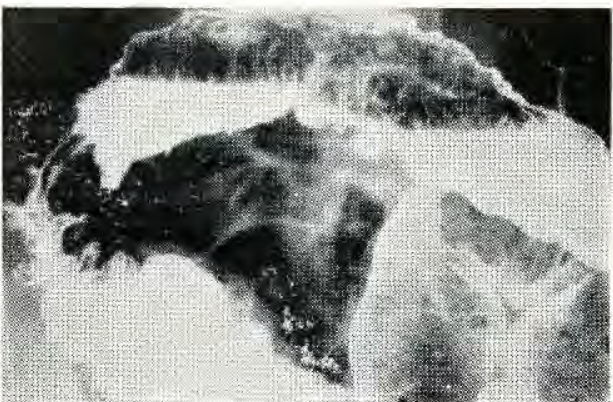

Fig. 6. Double contrast barium enema, erect view. Advanced Crohn's disease of the transverse colon, presenting a cobble-stone patlern. In the sigmoid colon numerous aphthous ulcerations are seen with normal intervening mucosa. 


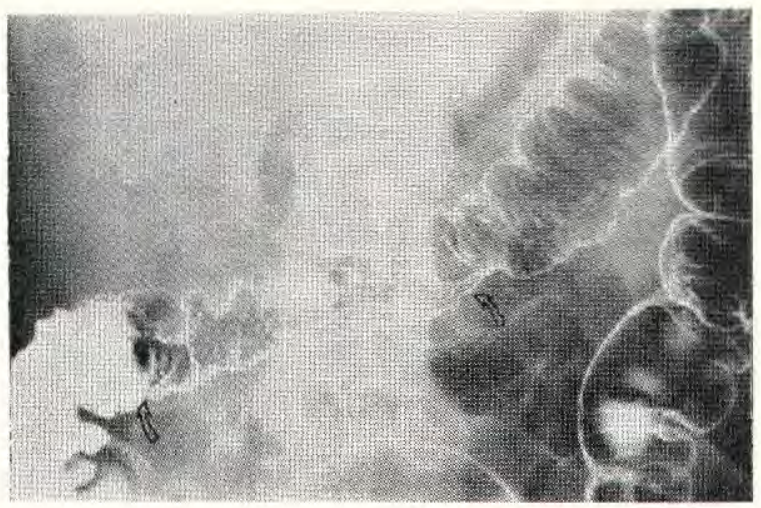

Fig. 7. Segment of Crohn's disease in the transverse colon. Hawstrations are normal and thickened on the upper border of the diseased segment and totally absent on the lower border. Normal colon distal to this area of disease,

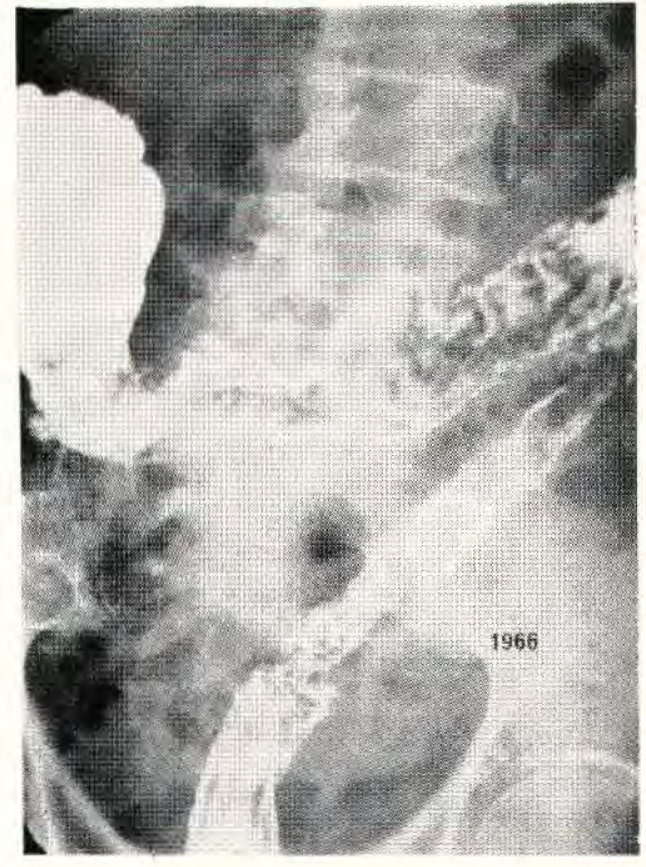

Fig. 8a. Barium enema 1966. Uneven involvement of the transverse and left colon by Crohn's disease.

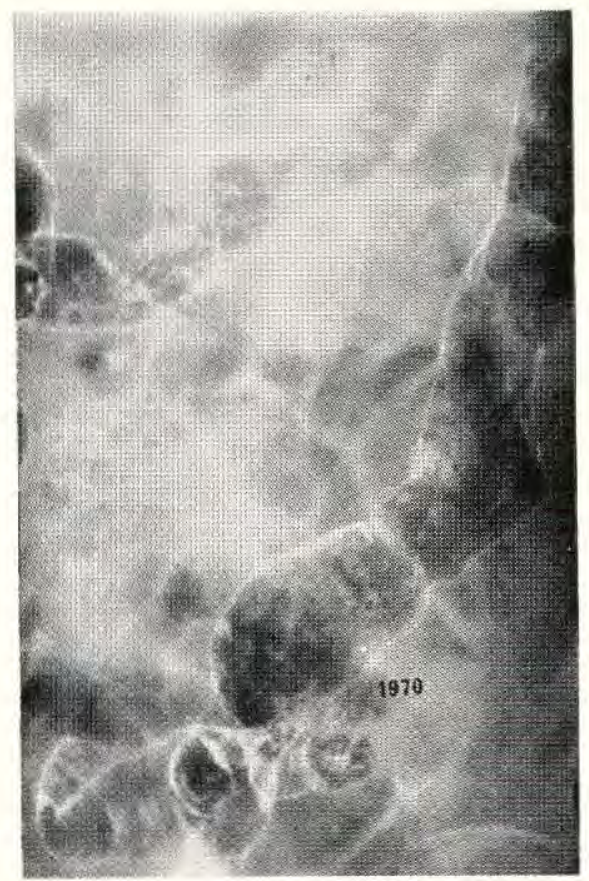

Fig. 8b. Re-examination four years later shows fistula formation between sigmoid colon and ileum.

The coexistence of Crohn's disease and diverticulitis is by no means rare. A second peak in the incidence of Crohn's disease occurs in the 50 to 60 year age group when diverticulitis is common. The normally fairly regular "picket fence", pattern in sigmoid diverticulitis is then altered to produce an uneven and interrupted pattern of lumen projections, often with ulceration. A typical finding may then be a barium filled track within the thickened bowel wall running parallel to the lumen of the gut (Figure 9). 


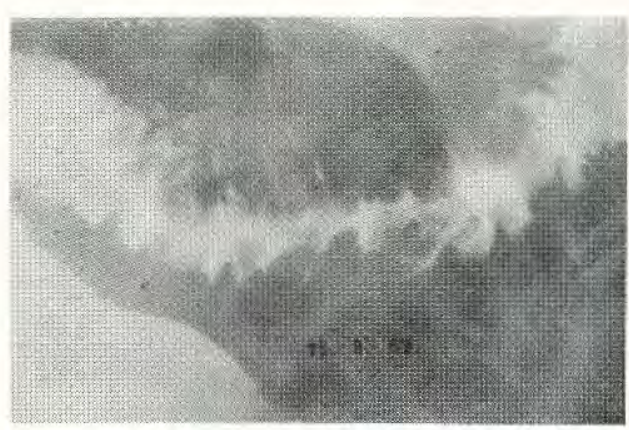

Fig. 9a. Barium enema, sigmoid colon. Diverticulitis with deeply penetrating projections of barium, somewhat irregularly arranged.

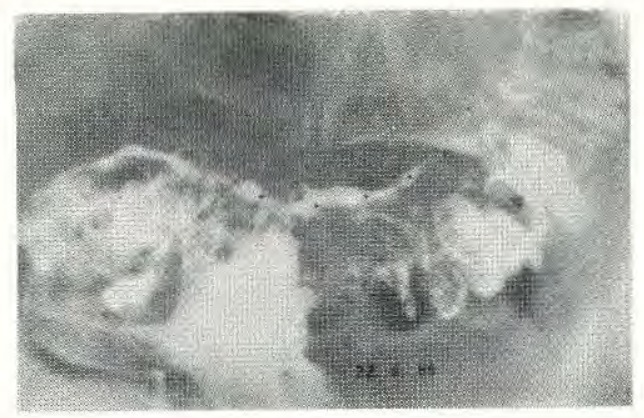

Fig $9 b$. Re-examination five weeks later. A mural, barium outlined track parallel to the lumen has developed along the upper border of the diseased sigmoid colon. Coexistence of diverticulitis and Crohn's disease.

\section{Small bowei Crohn's disease}

The usual follow-through of small bowel examination, i.e. the taking of $X$-ray films of the abdomen after the patient has swallowed a sufficiency of barium, has the capacity to demonstrate established and advanced Crohn's disease. A small bowel intubation technique has been developed which allows the more detailed study of the small intestine and makes possible the more exact demonstration of the upper limit of established Crohn's disease (Figure 10) and the delineation of segments of earlier disease proximal to and separate from the established distal disease. By the intubation method it is possible to demonstrate in the small bowel the same features of Crohn's disease which would be shown in the colon, i.e. linear ulcerations, nodule formation, aphthous ulceration, transaxial differentiation of intensity (Figure 11).

It may be important to distinguish in the distal ileum changes caused by an appendix abscess from those due to Crohn's disease with an inflammatory mass. The small bowel enema can do this well since it can differentiate between a primarily mucosal inflammatory process and one in which the mucosa is involved by extrinsic inflammation.

\section{Angiography in Crohn's disease and ulcerative colitis}

The pathognomonic angiographic feature of colonic Crohn's Disease is the "zoning" sign present in half the cases, consisting of an inner opacifying cylinder of gut wall and an outer layer with absent or diminished opacification (Figure 12). Generally, colonic Crohn's disease shows increasing arterial opacification of the thickened bowel wall with a fairly dense and uneven parenchmyal phase. The venous phase is intensified and slightly premature. Appearances in ulcerative colitis are totally different. There is rapid inflow of contrast medium and immediate outflow through mural veins, A parenchmyal phase barely develops. The zoning sign is not present.

Angiography has little practical application in the days of colonoscopy and the double constrast barium enema. There is still occasional indications for its use, particularly in older patients where the barium enema may not succeed. 


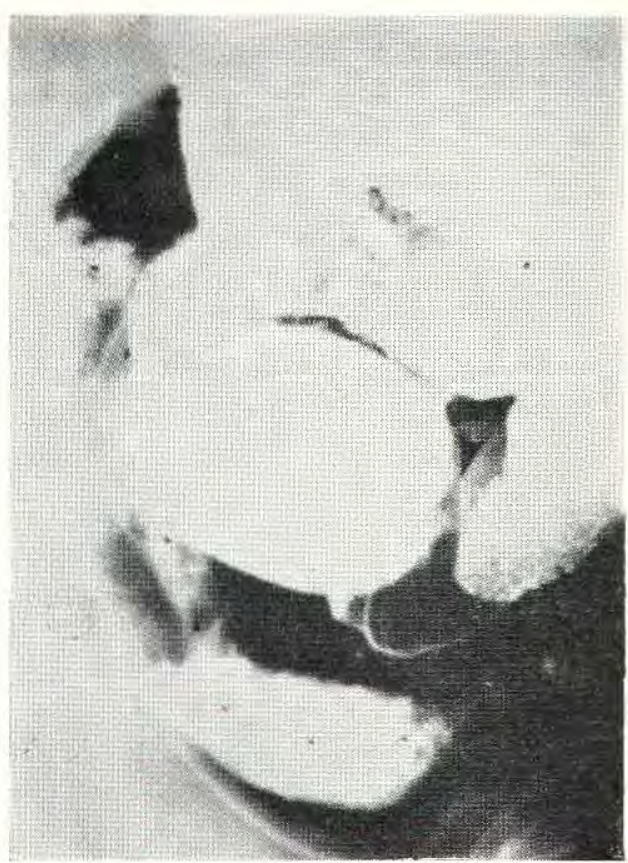

Fig. 10a. Follow-through examination of the small bowel shows established Croln's disease of the terminal ileum (dots).

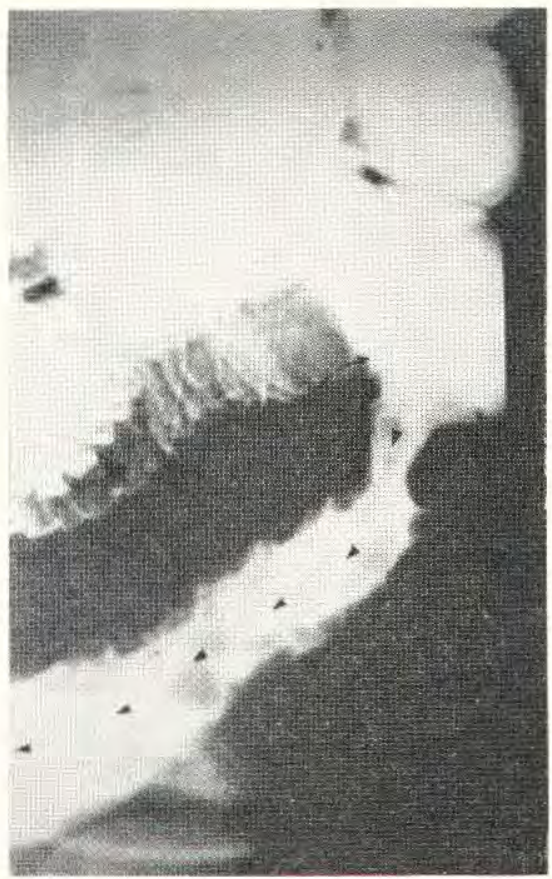

Fig 10b. Only the double contrast small bowel enema made it possible to demonstrate the exact upper limit of the disease process in the terminal ileum.

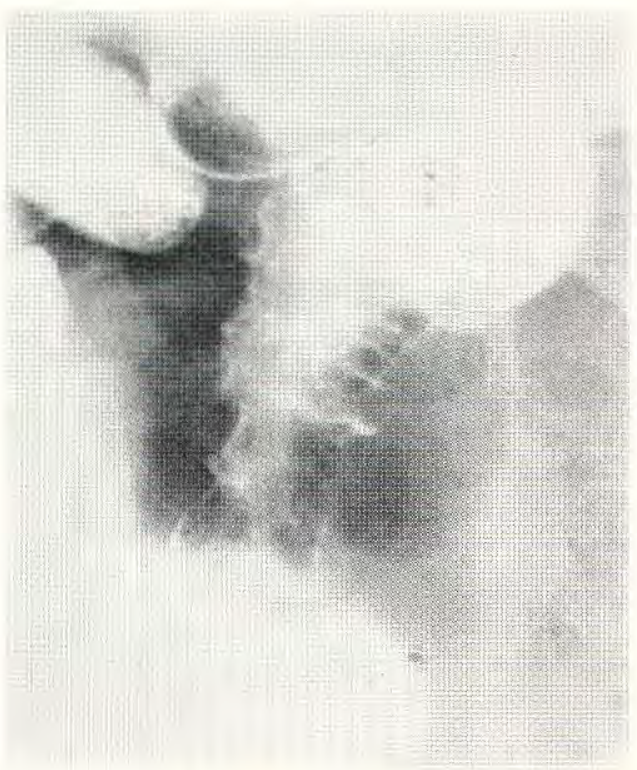

Fig. 11. Small bowel enema showing a segment of Crohn's disease in the upper ileum separate from established disease in the distal ileum. Transaxial linear ulcerations are clearly demonstrated. 


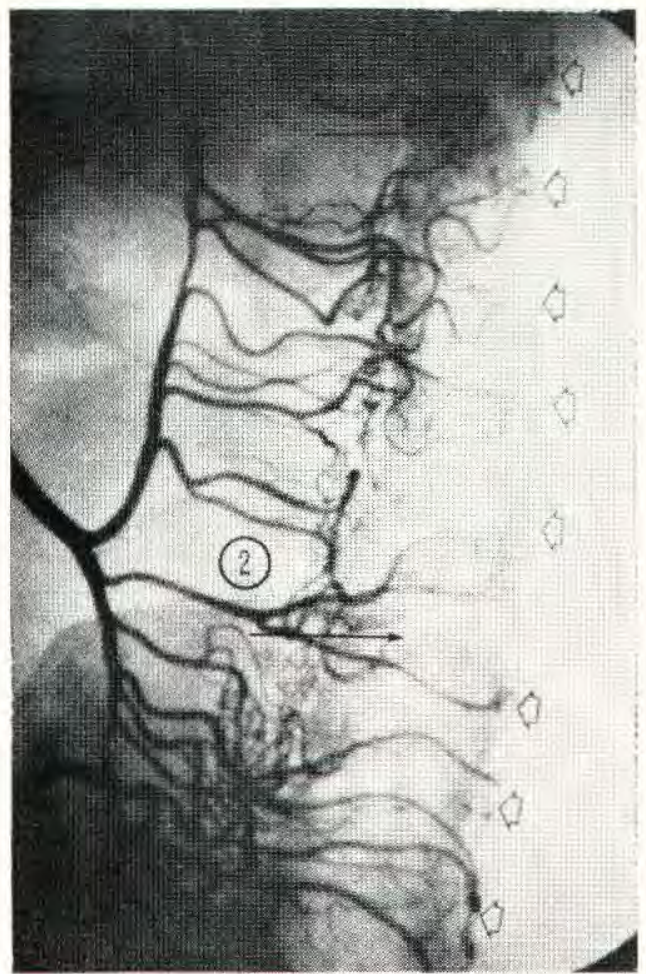

Fig. 12a. Inferior mesenteric arteriogram, arterial phase Crohn's disease of the descending colon. An inner cylinder of the thickened bowel wall shows incipient opacification while a poorly opacified outer layer is indicated by displacement of vasa recta terminations (arrows).

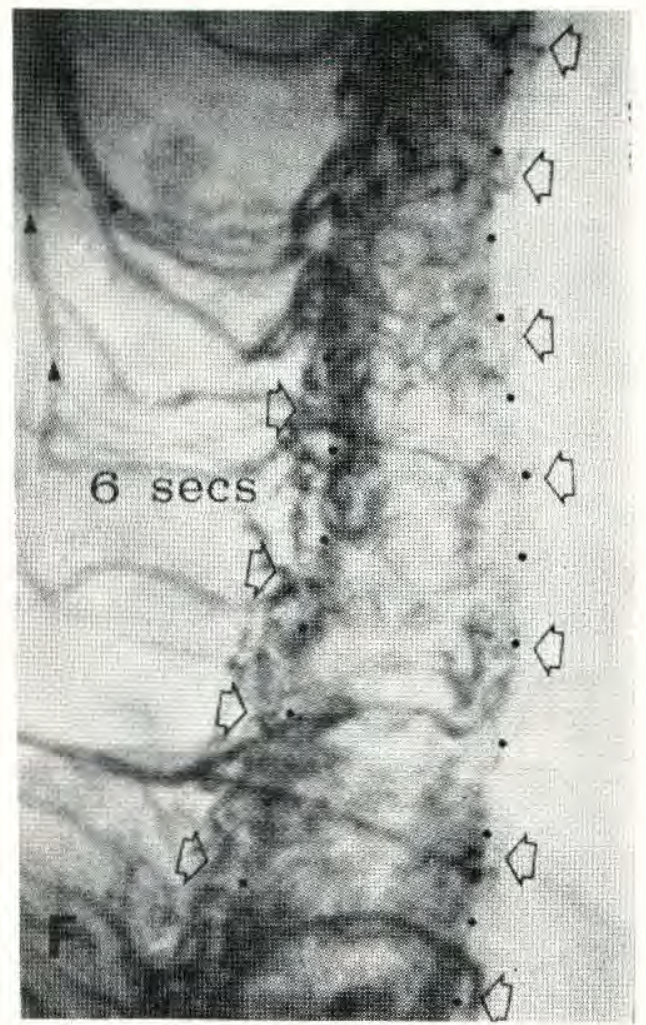

Fig 12b. Venous phase. Venae rectae origins are separated from the densely opacificd inner core of the descending colon. Positive "zoning sign" pathogonomonic of Crohis's disease in the colon.

\section{Order of St John of Jerusalem}

Notification has been received from the Secretary General of the Grand Priory in the British Realm of the Most Venerable Order of the Hospital of St John of Jerusalem that the following officers have been appointed Serving Officers of the Order.

\section{Officer Brother}

Maj Gen H A J Reay, QHP, MB, FRCP (Lond \& Ed). Col D Hamilton, MB, FRCP. Lt Col M S Owen-Smith, MS, MB. Lt Col W M Eider, TD, MB, ChB (TAVR). Lt Col P W Seargeant, TD, MB, FRCS (TAVR). Maj A T Merson, TD, MB, ChB (TAVR). Maj D H P Hopton, RADC. Capt A W S Spargo, RAMC. 